\title{
Strategic Flexibility Analysis in Emergency Medical Service Systems using Decision Rules
}

\author{
$1^{\text {st }}$ Yuan Zhou \\ Post-doctoral Research Station \\ China Guangfa Bank \\ Guangzhou, China \\ zhouyuan@cgbchina.com.cn
}

\author{
$2^{\text {nd }}$ Michel-Alexandre Cardin \\ Dept. of Industrial Systems Engineering and Management \\ National University of Singapore \\ Singapore, Singapore \\ macardin@nus.edu.sg
}

\author{
$3^{\text {rd }}$ Sizhe Zhang \\ Dept. of Data Science \\ Grab \\ Singapore, Singapore \\ sizhe.zhang@grab.com
}

\begin{abstract}
The capacity deployment of emergency medical service systems has been studied over decades. The design problem is still challenging due to the long-term uncertainty like the change of demographics. A novel approach in the form of multi-stage stochastic programming that considers flexibility in engineering design could be a valuable alternative to EMS infrastructure systems design. This paper proposes a hybrid algorithm to the stochastic model to improve its computational efficiency. The new algorithm outperforms other alternative methods in the numerical analysis by $3-6 \%$ in terms of quality of the solution, and is at least four times faster than the default methods.

Index Terms-flexibility in engineering design, emergency medical services, multi-stage stochastic programming, experimental algorithm, metaheuristics
\end{abstract}

\section{INTRODUCTION}

Emergency medical service (EMS) systems play a significant role in the urban infrastructure systems, which aims to provide quick out-of-hospital medical treatment to patients and transport them to the nearest hospital if necessary. It has been demonstrated that efficient EMS systems could largely increase the patients' survival rates through delivering fast defibrillation [1]. However, managing EMS systems can be challenging in both short-term and long-term, especially in developing countries. This is because in developing countries, such as India and China, uncertainty like the changes in demographics over time is fairly fast as compared to developed countries. This long term uncertainty inevitably influences the overall need for the EMS systems, and also has an impact on the incident patterns. Hence, an EMS system which was designed based on the short term uncertainty may easily become sub-optimal in the long term.

More specifically, an EMS system deployed in view of "average" or "most likely" scenario prediction may perform badly in the reality due to a phenomenon known as "Flaw of Average" [2]. To tackle this issue, and to pro-actively deal with uncertainty, Reference [3] proposed an approach to manage the system's capacity and configuration in the face of future possibilities. The method is built upon the idea of flexibility in engineering design, which provide the "right, but not the obligation, to change the system easily in the face of uncertainty" [4]. In contrast to typical EMS systems, the flexible design enables the novel EMS system to be adaptable to the future possibilities by gradually deploying capacity over time and space. This proposed EMS system is developed via a multi-stage stochastic model in consideration of long term uncertainty. The computational complexity may be a potential issue when the scale of the design problem is considerably large.

To address the concern mentioned above, a hybrid algorithm is proposed in this article. The algorithm aims to solve the optimization problem in a relatively shorter amount of time, while the quality of the solution should be maintained in an acceptable level. For the purpose of demonstration, a case study is conducted in the context of a hypothetical city in an emergency country. The input data is synthetic, which derived from the real data from a published study.

This paper is organized as follows. Section 2 presents a short literature review, containing the related works in capacity deployment of EMS systems, flexibility and real options analysis, and algorithms for multi-stage stochastic programming. Section 3 explicitly describes the formulation of the mathematical model and its sub-problems, as well as the proposed hybrid algorithm. Section 4 illustrates the comparison between the hybrid algorithm and default method via a case study. Concluding remarks and limitations are presented in Section 5.

\section{Literature REVIEW}

The literature review consists of three parts. The first two subsections introduce the background knowledge of the novel approach used for capacity deployment in EMS systems, while the last one discusses the possible algorithms for solving the multi-stage stochastic programming model efficiently.

\section{A. Capacity Deployment of Emergency Medical Service (EMS) Systems}

The design of EMS systems has been received attentions in recent decades. The literature is fairly extensive (e.g., [5], [6]) and mainly focuses on capacity planning and operations (a.k.a resource allocation-reallocation problem). This allocation problem could be categorized into two types: maximal covering location problem (i.e., MCLP) and set covering location problem (i.e., SCLP). The former aims to maximize the population covered by the limited emergency resources (e.g., emergency vehicles), while the objective of the latter is 
to minimize the resource used for fulfilling the predetermined request. Both types of problems have been studied for many years, and various methods like queuing theory, probabilistic constraints, and stochastic optimization are applied to such problems to deal with uncertainty [7]-[9].

However, there is not much research accounting for capacity deployment in a relatively longer periods with consideration of uncertainty. According to the reviews, most of the works are related to the short-term planning under uncertainty. Reference [10] studied dynamic version of MCLP under the assumption of deterministic emergency requests. Reference [11] analyzed a multi-allocation problem regarding a single facility. Little research has considered using flexibility to tackle the uncertainty in EMS systems, except for [3], which developed a multi-stage stochastic programming model incorporating the flexibility and managerial decision rules to deploy emergency resource over time and space.

\section{B. Flexibility in Engineering Design (Real Options)}

Flexibility in engineering design is a promising approach to the capacity deployment of EMS systems by enabling the system to change itself to adapt to the future possibilities. Reference [12] proposed a five-phase taxonomy and design framework for systematically incorporating the flexibility in complex engineering systems. The flexibility, or real options, typically falls into two groups: real options "in" projects and real options "on" project. Real options "in" project requires an in-depth understanding of the targeted system so that one can make decisions such as staging deployment of capacity and upgrading/downgrading capacity over time. In contrast, real options "in" projects normally treats the system as a black box and focuses on high level decision-making like continuing or deferring investment based on the market performance.

It has been demonstrated that flexibility in design could improve the system performance by $10-30 \%$ in terms of the expected life cycle costs in various industrial applications [13]. It, however, should be noted that flexibility may not always be favorable because of the high cost premium and the setup of the "benchmark". If the economies of scale are particularly strong (implying that deploying more capacity at once is considerably cheap), or the discount rate is fairly low (implying that investing now is more or less equal to investing later), then there is little benefit to defer the capacity deployment cost. As demonstrated in the paper, though, flexibility is still valuable in the context of EMS systems.

\section{Algorithm for Multi-stage Stochastic Programming}

Solving multi-stage stochastic programming (MSP) is challenging in general. However, the model could be solvable if the formulation is well structured (e.g., scenario tree). This structure requires reliable sampling methods to generate representative samples, so that the solution based on these samples can be robust to other unseen scenarios. Commonly used sampling approaches are Monte-Carlo simulation via Sample Average Approximation (SAA) method [14], Markov Chain Monte Carlo (MCMC) [15], etc.
To efficiently solve the tree structure MSP models, decomposition is the one of the basic and fundamental algorithm. Benders decomposition (a.k.a cutting-plane approach), Dantzig-Wolfe decomposition, and nested L-shaped decomposition, respectively, are typical decomposition methods for solving very large linear programming problems with special bock structure (e.g., MSP). Specifically, Benders decomposition splits the original problem into two parts: the firststage master problem and the second-stage sub-problems. The values for decision variables in the sub-problems are determined by a given first-stage solution [16]. If the subproblem is determined to be infeasible due to the given firststage solution, a few rows will then be generated and added to the master problem. In contrast, Dantzig-Wolfe decomposition will generate columns and add to the master problem under such situation [17]. Nested L-shaped decomposition in fact breaks down multi-stage to two-stage sub-problems and then repeatedly applies L-shaped method to the blocks of each twostage sub-problem.

Besides the above algorithms, metaheuristics could also used for solving MSP problems, such as Ant Colony Optimization, Evolutionary Computation, Simulated Annealing, and Tabu Search. As compared to exact methods like Branch and Bound, metaheuristics is acceptable in terms of the quality of the solution, while the computational time is much less than exact methods when the scale of the problem is fairly large.

\section{The Hybrid Algorithm}

This section introduces the mathematical formulation of the multi-stage stochastic programming model, and also explicitly illustrates the hybrid algorithm for efficiently solving such problem. This resource allocation model is basically presented in [3]. For simplicity, only the general form is discusses in this paper.

\section{A. Multi-stage Stochastic Programming Model}

The objective of the MSP model is to minimize the expected total cost over the system's life cycle, subject to limited emergency resources. Hence, it can be considered as an extension of SCLP. In general, the model is formulated as follows, based on the sample average approximation assumption:

$$
\begin{gathered}
\min _{x^{1} \in \chi^{1}} f^{1}\left(x^{1}\right)+\sum_{s=1}^{S} p_{s} Q_{s}^{2}\left(x^{1}, \zeta_{s}^{[2]}\right) \\
\text { s.t. } Q^{t}\left(x^{t-1}, \zeta^{[t]}\right)=\inf _{x^{t} \in \chi^{t}\left(x^{t-1}, \zeta^{t}\right)}\left[f^{t}\left(x^{t}, \zeta^{t}\right)\right. \\
\left.+\sum_{s=1}^{S} p_{s} Q_{s}^{t+1}\left(x^{t}, \zeta_{s}^{[t+1]}\right)\right], t=2, \ldots, T ; \\
x_{k}^{t}=x_{l}^{t}, k \neq l, \zeta_{k}^{[t]}=\zeta_{l}^{[t]}, k, l \in S, t \in T .
\end{gathered}
$$

The MSP model consists of $S$ scenarios in a tree structure. Decision variable $x^{t}$ is determined by observations up to period $t$. Hence, if the observations for two separate scenarios are exactly the same, the model should have the same output 
(which is $x$ ). This is the so-called nonanticipative constraint (see (3)). Equation (2) is the form of sub-problems backward from last period $T$. It represents that the model minimizes the objective value at each period recursively. More details of this model can be found in [3].

\section{B. Algorithm}

To efficiently solve Problem (1)-(3), a hybrid algorithm consists of two phases (i.e., inner and outer search) is proposed. The structure of the algorithm can be found in Fig.1. As can be seen, one may initially determine a basic feasible solution (BFS) for the outer search, and then find the corresponding optimal solution for capacity deployment based on this BFS via inner search. The final solution is calculated based on solutions from both inner and outer search. The whole procedure will be terminated once the stopping criteria are triggered (either time and/or iteration limit).

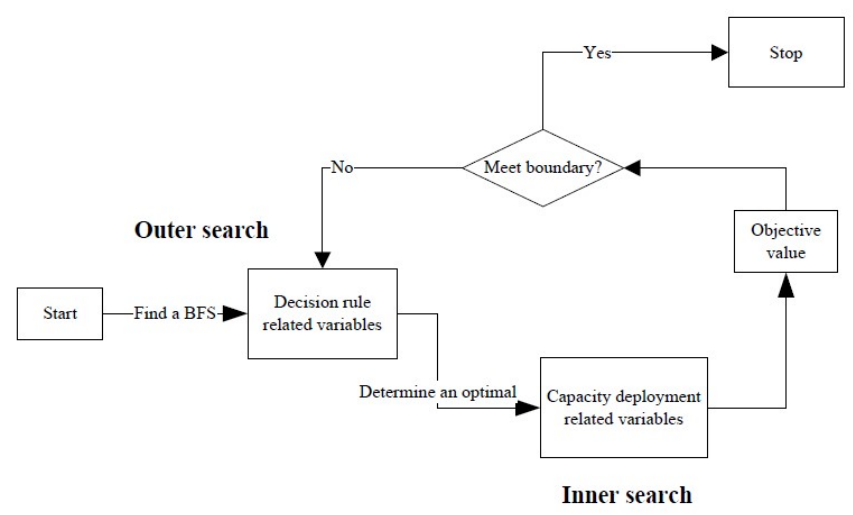

Fig. 1. Flow chart for the two-phase hybrid algorithm

This hybrid algorithm requires to dividing the original problem into $S$ sub-problems as they are supposed to be independent. The sub-problem can then be formulated as:

$$
\begin{gathered}
\min _{x^{1} \in \chi^{1}} f^{1}\left(x^{1}\right)+Q_{s}^{2}\left(x^{1}, \zeta_{s}^{[2]}\right) \\
\text { s.t. } Q^{t}\left(x^{t-1}, \zeta^{[t]}\right)=\inf _{x^{t} \in \chi^{t}\left(x^{t-1}, \zeta^{t}\right)}\left[f^{t}\left(x^{t}, \zeta^{t}\right)\right. \\
\left.+Q_{s}^{t+1}\left(x^{t}, \zeta_{s}^{[t+1]}\right)\right], t=2, \ldots, T
\end{gathered}
$$

where $x^{1}$ in (4) is a given value rather than a decision variable. As can be seen, Problem (4)-(5) is an integer programming. These sub-problems will be resolved by Branch and Bound, which is used as inner search method of the hybrid algorithm.

In addition to solving the sub-problems, another important part is to find an optimal $x^{1}$. It is anticipated that the combination of parameters is considerably large, and thus an exhaustive search is impractical. An experimental approach called Adaptive One-Factor-at-a-Time (aOFAT) is applied to deal with this challenge. Fig.2 illustrates the process of this aOFAT method. Unlike typical OFAT, the interactions between different factors are considered in this adaptive method. It is assumed that there are $n$ factors, and that there is a response $y$ which is a function of the factors (e.g., objective function). Furthermore, it is assumed that the factors have two levels each, coded as $x_{i} \in\{-1,1\}$, yet it can be more levels in practice. The initial point (or the baseline observation) could be $O_{0}=y\left(\tilde{x_{1}}, \tilde{x_{2}}, \ldots, \tilde{x_{n}}\right)$. After the first step, $x_{1}$ is toggled and we have the corresponding observation $O_{1}=y\left(-\tilde{x_{1}}, \tilde{x_{2}}, \ldots, \tilde{x_{n}}\right)$. The final value of $x_{1}$ will be determined by taking the difference between $O_{0}$ and $O_{1}$, and is denoted as $x_{1}^{*}$.

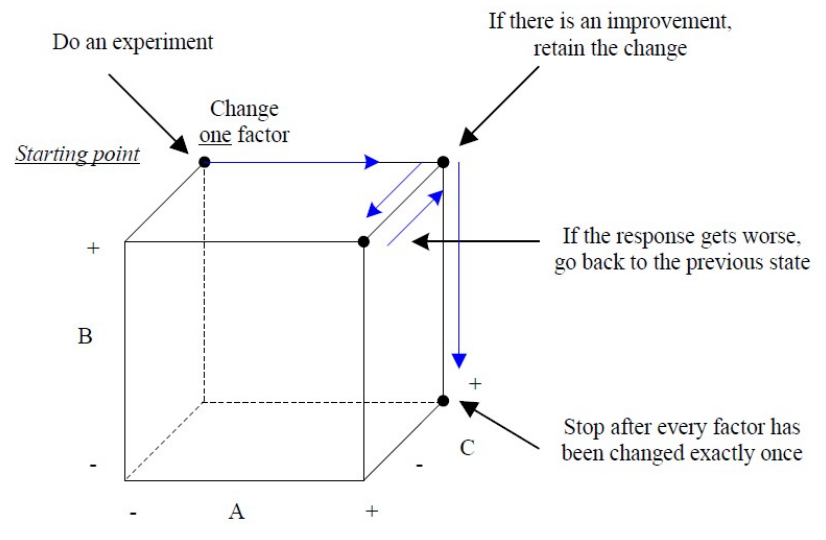

Fig. 2. Visualization of Adaptive OFAT [18]

Note that none of these interactions are exploited after this step. However, all of the factors except for $x_{1}$ are toggled in the subsequent steps of the aOFAT; their final state may be different from the state after the first step. In that case, the contributions due to interactions $\beta_{1 j}$ may potentially be reversed as the process continues. In contrast, the first main effect $\beta_{1}$ is exploited and its contribution is permanent. In the second step, we toggle $x_{2}$ to see whether there is improvement in the observation. The interactions $\beta_{12}$ will not be affected in any way by subsequent experiments. The probability of exploiting the interaction $\beta_{12}$ will be greater than $50 \%$ for all systems with nonzero interactions [18], which is better than that provided by random chance. If $\beta_{12}$ is the largest interaction, this probability is no less than $75 \%$. Reference [18] proved that the probability of exploiting any interaction $\beta_{i j}$ is no less than the probability of exploiting $\beta_{12}$.

\section{NUMERICAL ANALYSIS}

This section discusses the performance of the proposed hybrid algorithm via a numerical analysis. This design problem is based on a hypothetical city, consisting of ten districts. The objective of the design problem is to minimize the expected total costs over system's life cycle (which is ten years). The strategic decisions - such as capacity deployment of emergency facilities and purchase of emergency vehicles - 
are determined every year, while the tactical decisions - such as allocating and reallocating emergency vehicles - are decided every quarter. To formulate the MSP model, about twenty representative sample scenarios are generated via Geometric Brownian Motion (GBM) process. The formula of the GBM is like this:

$$
d n_{j, t}=\mu n_{j, t} d t+\delta n_{j, t} d W_{t} .
$$

$W_{t}$ is a Wiener process or so-called Brownian motion, while $\mu$ and $\delta$ are, respectively, the expected growth rate and volatility derived from historical data. $n_{j, t}$ denotes the uncertainty (e.g., incident arrival rate) on site $j$ at state $t$. Generating samples via GBM is kind of reasonable since the drift (i.e.,mean growth rate) and the random shock (i.e., volatility) could well represent the future possibilities of emergency incidents along with the change of demographics. More details about the case study can be found in [3].

The hybrid algorithm is compared to the default Branch and Bound (B\&B) method, which is considered as the benchmark. Moreover, Simulated Annealing (SA) and Genetic Algorithm (GA, as an example of evolutionary algorithm) are taken into account as the alternative algorithms for the outer search of the hybrid algorithm. All outer search heuristics were coded in AIMMS 4.2 and CPLEX 12.6 was selected as the solver for implementing the inner search and the benchmark. All alternative methods were run on the same high performance workstation with $2.60 \mathrm{GHz} \mathrm{CPU}$ and $32 \mathrm{~GB}$ RAM to make the comparison fair enough. The comparison focuses on two metrics of interest: Quality of Solution $(\mathrm{QoS})$ and Time-toBest-Solution (TBS).

\section{A. Quality of Solution}

The quality of solution is defined as the relative gap between the best solution of the optimization problem obtained from the algorithm and the best LP bound. For linear programming, the best solution should be the same as the best LP bound if it claims to be the optimal. In this numerical analysis, the best LP bound is determined by the $\mathrm{B} \& \mathrm{~B}$ method in the commercial solver (i.e., CPLEX) and thus it is reliable. It is known that the smaller the gap is, the better the quality of the solution.

Fig. 2 shows a comparison of the results of four runs across different incident coverage rates (fleet size). The solution consists of initial configuration and decision rules parameters (i.e., $\left.x^{1}\right)$. As can be seen, the values of final solutions for all alternative methods, and the best LP bound, are increasing, along with the increase of coverage rate. This makes sense because to achieve a higher incident coverage rate the system definitely needs more budget. However, the increment of the budget may not necessarily need to be consistent with the same increment in coverage rate. That is, the $\Delta B$ ( $B$ denotes the objective value) is not the same for coverage rate increasing from 0.85 to 0.9 and from 0.9 to 0.95 .

As observed in the figure, the hybrid heuristic is generally the best of all four alternatives in terms of the gap between the solutions and bounds, while the benchmark algorithm performs the worst in all cases. This may be because the standard B\&B

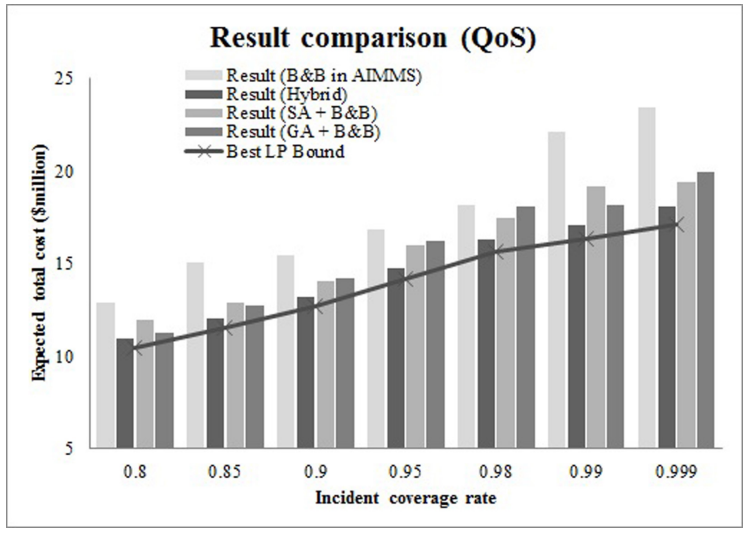

Fig. 3. Comparison of quality of solution across different coverage rates

algorithm is too general and is thus not well suited for this type of design problem (and its formulation). Besides, the performances for the other two metaheuristics are roughly the same when the coverage rate is between $85 \%$ and $98 \%$, and both are a bit worse than the hybrid algorithm. This may be due to the mechanism of SA and GA, since they are based on random search and thus no initial treatment as there is for first-stage variables in the hybrid method. The quality of the solution, therefore, heavily depends on the number of iteration limits defined initially; in general, the larger the number is, the better the quality for SA and GA.

\section{B. Time to Best Solution}

This metric is the exact CPU solving time subjected to the iteration limits (i.e., four million iterations) or relative gap limits (i.e., $1 \%$ gap), whichever reaches the first. The default upper bound of the solving time is 100,000 seconds (i.e., approximately one day and four hours), which may not be acceptable in practice.

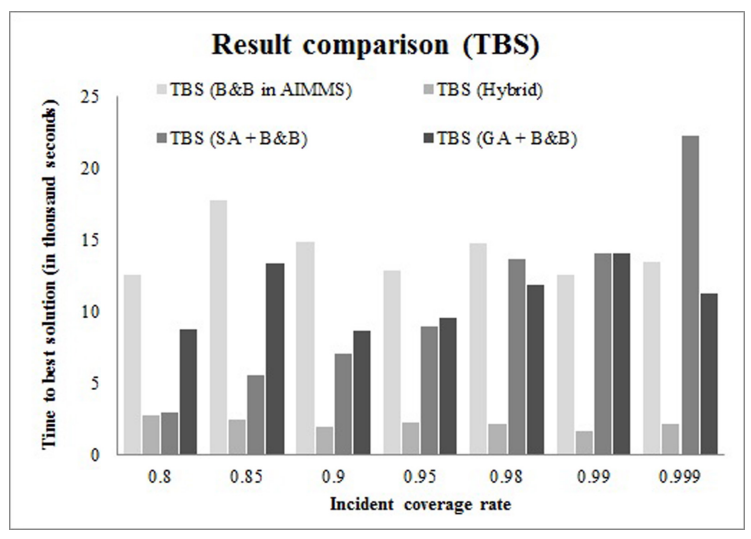

Fig. 4. Comparison of time-to-best-solution across different coverage rates

Fig. 3 shows the comparison result for the four methods. Overall, it indicates that the hybrid algorithm is generally faster than the other methods. More specifically, the solving time of the hybrid algorithm never goes beyond 3,000 seconds. Only the solving time of SA method in the scenario of $80 \%$ 
coverage rate can be close to this value (i.e., 2,980.47 seconds). Generally, metaheuristics can find the "good enough" solution relatively quicker than the benchmark method. However, when the coverage rate is greater than $98 \%$, the benchmark seems faster than SA and GA. This may be due to the drawback of the random search. When the constraint is tighter, the set of feasible solution for the benchmark is relatively smaller than metaheuristics and thus the standard B\&B is more efficient.

One interesting point is that SA costs less than GA when coverage rate is relatively small (e.g., 95\%), and it costs more in the reverse cases. Furthermore, only the TBS for SA increases alongside the coverage rate. This makes sense as SA searches the neighborhood solution in every iteration, while the solving time for each solution increases with the coverage rate. For GA, the time is not consistently increasing due to the randomness of making crossovers between the generated solutions and mutating new solutions. Although the solving time is not dependent on the coverage rate and thus intractable, the hybrid method is still applicable in practice as the absolute amount of time is relatively small and fairly stable.

\section{Out-of-Sample Test}

This purpose of this out-of-sample test is to ensure that the solutions obtained from heuristics are as reliable as those obtained from the commercial solver. Unseen scenarios (to model) are used as testing samples to evaluate the quality of the optimal solution (if any) or the "good enough" solutions. Fig.4 represents that the hybrid method outperforms the other methods in general, in terms of the expected total costs over those unseen samples.

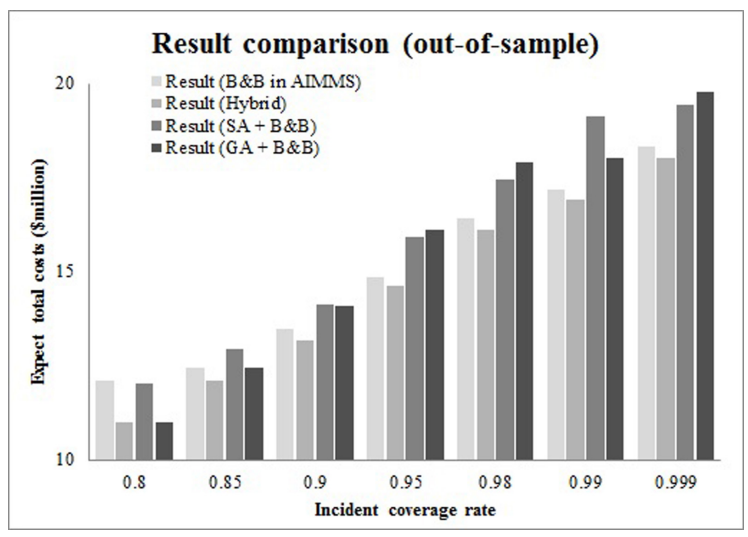

Fig. 5. Comparison of out-of-sample tests across different coverage rates

The benchmark method is the second best, outperforming $\mathrm{SA}$ and GA when the coverage rate is higher than or equal to $90 \%$. Although the difference between the objective values of the hybrid method and the standard B\&B method generally becomes smaller, the solution of the hybrid method is still more reliable than that of the benchmark method, according to the statistical analysis of the out-of-sample test (see Table I). As can be seen, the hybrid method outperforms the others in all metrics. The difference between the mean values for the hybrid and benchmark is statistically significant, in terms of its $p$-value $(0.0029 \ll 0.05)$. Although the standard $\mathrm{B} \& \mathrm{~B}$ is the second best, its standard deviation (1.07) is the largest. This may be because this method doesn't solve the sub-problems to optimally, as compared to other methods. Again, the out-ofsample test shows that the hybrid method yields more reliable solutions than others.

TABLE I

SUMMARY OF THE STATISTICAL ANALYSIS

\begin{tabular}{|c|c|c|c|c|c|c|}
\hline Method & Mean & STD & STD error & P5 & P50 & P95 \\
\hline B\&B & 13.48 & 1.07 & 0.034 & 11.86 & 13.39 & 15.31 \\
\hline Hybrid & 13.19 & 0.91 & 0.029 & 11.80 & 13.15 & 14.73 \\
\hline SA & 14.13 & 1.00 & 0.032 & 12.44 & 14.10 & 15.84 \\
\hline GA & 14.08 & 0.98 & 0.031 & 12.56 & 13.58 & 15.80 \\
\hline Best? & Hybrid & Hybrid & Hybrid & Hybrid & Hybrid & Hybrid \\
\hline
\end{tabular}

\section{Conclusions}

The typical resource allocation problem in the context of EMS system is considered as NP-complete problem, since the size of the solution space for locating $v$ emergency facilities/vehicles in $i$ districts is $i^{v}$ [19]. The complexity of this combinational nature requires various attempts to explore and exploit near-optimal solutions for practical use. This paper aims to propose a hybrid algorithm to solve an even more complex problem, which considers both long-term uncertainty and flexibility in engineering design during the development. The proposed two-phase framework outperforms the standard Branch and Bound algorithm in the numerical analysis, as well as other metaheuristics, in terms of quality of solution and time-to-best-solution. The analysis also demonstrates that the solving time of the proposed hybrid method doesn't increase along with the increase of the scale of the problem, which makes it applicable for large scale design problems.

Still, there is much room for further research. First of all, both inner and outer search could be improved by exploring other algorithms. For now, the inner search is standard B\&B method, while the outer search is based on an experimental approach. Effective heuristics could be developed for inner search to shorten the solving time when the scale is fairly large. Also, more (meta)heuristics and decomposition methods may be worthy exploring to improve the efficiency of the outer search. Secondly, besides the metrics discussed in this paper, other metrics such as convergence rate and memory used for the algorithm. The former theoretically tells us whether the algorithm could converge to a stable value in an acceptable amount of time or not, while the latter shows the applicability of the algorithm. Last but not the least, it is possible to test a few more numerical cases to demonstrate the generality of the method.

\section{ACKNOWLEDGMENT}

This work was supported by the Post-doctoral scholarship. The authors are also thankful for the feedback provided on this work by Bing Wang and Ping Xie. 


\section{REFERENCES}

[1] M.E. Ong, F.S. Ng, J. Overton, S. Yap, D. Andresen, D.K. Yong, S.H. Lim, V. Anantharaman, "Geographic-Time Distribution of Ambulance Calls in Singapore: Utility of Geographic Information System in Ambulance Deployment (CARE 3)," Ann Acad Med Singapore, vol. 38, pp. 184-191, March, 2009.

[2] S. Savage, "Flaw of Averages," Harvard Bus. Rev. 4.

[3] S. Zhang, M.A. Cardin, "Flexibility and real options analysis in emergency medical services systems using decision rules and multi-stage stochastic programming," Transp. Res. Part E: Logist. Transp. Rev., vol. 107, pp. 120-140, November, 2017.

[4] L. Trigeorgis. Real Options: Managerial Flexibility and Strategy in Resource Allocation. MIT Press, Cambridge, MA, 1996.

[5] X. Li, Z. Zhao, X. Zhu, T. Wyatt, "Covering models and optimization techniques for emergency response facility location and planning: a review," Math. Methods Oper. Res., vol. 74, pp. 281-310, December, 2011.

[6] R.Z. Farahani, N. Asgari, N. Heidari, M. Hesseinina, M. Goh, "Covering problems in facility location: a review," Comput. Ind. Eng., vol. 62, pp. 368-407, February, 2012.

[7] D. Shishebori, A. Yousefi Babadi, "Robust and reliable medical services network design under uncertain environment and system disruptions," Transp. Res. Part E: Logist. Transp., vol. 77, pp. 268-288, May, 2015.

[8] A.Y. Chen, T.-Y. Yu, "Network based temporary facility location for the Emergency Medical Services considering the disaster induced demand and the transportation infrastructure in disaster response," Transp. Res. Part B: Methodol., vol. 91, pp. 408-423, September, 2016.

[9] Y. Liu, Z. Li, J. Liu, H. Patel, "A double standard model for allocating limited emergency medical service vehicle resources ensuring service reliability," Transp. Res. Part C: Emerg. Technol., vol. 69, pp. 120-133, August, 2016.

[10] R.Z. Farahani, Z. Drezner, N. Asgari, "Single facility location and relocation problem with time dependent weights and discrete planning horizon,” Ann. Oper. Res., vol 167, pp. 353-368, March, 2009.

[11] G. Gunawardane, "Dynamic versions of set covering type public facility location problems," Eur. J. Oper. Res., vol 10, pp. 190-195, June, 1982.

[12] M.A. Cardin, "Enabling Flexibility in Engineering Systems: A Taxonomy of Procedures and a Design Framework," J. Mech. Des., vol 136, pp. 011005-011005-14, November, 2013.

[13] R. de Neufville, S. Scholtes. Flexibility in Engineering Design. MIT Press, Cambridge, MA, 2011.

[14] A. Shaprio. "Sample Average Approximation," in Encyclopedia of Operations Research and Management Science, S.I. Gass and M.C. Fu, Eds. Boston, MA: Springer, 2013, pp. 1350-1355.

[15] R.L. Smith, "Efficient Monte Carlo Procedures for Generating Points Uniformly Distributed Over Bounded Regions", Oper. Res., vol 32, pp. 1296-1308, November 1984.

[16] J.F. Benders, "Partitioning procedures for solving mixed-variables programming problems", Numerische Mathematik, vol 4, pp. 238-252, December 1962.

[17] G.B. Dantzig, P. Wolfe, "Decomposition Principle for Linear Programs", Oper. Res., vol 8, pp. 101-111, February 1960.

[18] D.D. Frey, H. Wang, "Adaptive One-Factor-at-a-Time Experimentation and Expected Value of Improvement", Technometrics, vol 48, pp. 418431, 2006

[19] C. Saydam, J. Repede, T. Burwell, "Accurate estimation of expected coverage: A comparative study", Socio-Econ. Plan. Sci., vol 28, pp. 113-120, 1994. 